\title{
Article \\ A Heuristic Projection Pursuit Method Based on a Connection Cloud Model and Set Pair Analysis for Evaluation of Slope Stability
}

\author{
Mingwu Wang ${ }^{1, * \mathbb{D}}$, Yan Wang ${ }^{2, * \mathbb{C}}$, Fengqiang Shen ${ }^{1} \mathbb{D}$ and Juliang Jin ${ }^{1}$ \\ 1 School of Civil and Hydraulic Engineering, Hefei University of Technology, Hefei 230009, China; \\ shenfq@hfut.edu.cn (F.S.); $2000800040 @$ hfut.edu.cn (J.J.) \\ 2 College of Computer Science, Nankai University, Tianjin 300350, China \\ * Correspondence: mingwu_wang@hfut.edu.cn or wanglab307@foxmail.com (M.W.); \\ wangy@nbjl.nankai.edu.cn (Y.W.); Tel.: +86-551-62905718 (M.W.); Fax: +86-551-62902066 (M.W.)
}

Citation: Wang, M.; Wang, Y.;

Shen, F.; Jin, J. A Heuristic Projection

Pursuit Method Based on a Connection

Cloud Model and Set Pair Analysis for Evaluation of Slope Stability. Symmetry 2021, 13, 2016. https:// doi.org/10.3390/sym13112016

Academic Editors: José Carlos R. Alcantud and László T. Kóczy

Received: 17 September 2021

Accepted: 19 October 2021

Published: 24 October 2021

Publisher's Note: MDPI stays neutral with regard to jurisdictional claims in published maps and institutional affiliations.

Copyright: () 2021 by the authors. Licensee MDPI, Basel, Switzerland. This article is an open access article distributed under the terms and conditions of the Creative Commons Attribution (CC BY) license (https:// creativecommons.org/licenses/by/ $4.0 /)$.

\begin{abstract}
Determining the projection direction vector (PDV) is essential to the projection pursuit evaluation method for high-dimensional problems under multiple uncertainties. Although the PP method using a cloud model can facilitate interpretation of the fuzziness and randomness of the PDV, it ignores the asymmetry of the PDV and the fact that indicators are actually distributed over finite intervals; it quickly falls into premature defects. Therefore, a novel PP evaluation method based on the connection cloud model (CCM) is discussed to remedy these drawbacks. In this approach, adaptive numerical characteristics of the CCM are adopted to represent the randomness and fuzziness of the candidate PDV and evaluation indicators. Meanwhile, to avoid complex computing and to accelerate the convergence speed of the optimization procedure, an improved fruit fly optimization algorithm (FOA) is set up to find the rational PDV. Alternatively, candidate PDVs are mutated based on the mechanism "pick the best of the best" using set pair analysis (SPA) and chaos theory. Furthermore, the applicability and reliability are discussed based on an illustrative example of slope stability evaluation and comparisons with the neural network method and the PP evaluation method based on the other FOAs and the genetic algorithm. Results indicate that the proposed method with simpler code and quicker convergence speed has good global ergodicity and local searching capabilities, and can better explore the structure of high-dimensional data with multiple uncertainties and asymmetry of the PDV relative to other methods.
\end{abstract}

Keywords: projection pursuit; fruit fly optimization algorithm; metaheuristics; connection cloud model; set pair

\section{Introduction}

The optimization of high-dimensional problems has been a focal issue of computer science, artificial intelligence, management decision making, and engineering applications. Previously, the confirmatory data analysis (CDA) method has been used, with some assumptions of the data structure or distribution characteristics, and following specific criteria [1] — such as how the reliability analysis method and multivariate analysis method obey normal distribution. However, high-dimensional nonlinear problems are of multiple uncertainties and non-normal distribution characteristics. Consequently, the nonlinear problems of non-normal distribution or small-size samples under numerous uncertainties cannot be solved by the CDA method because they cannot meet the assumed conditions. In addition, evaluation methods based on classification criteria or empirical rules are widely used in practical engineering problems. Still, they have limitations in assessing problems under uncertain environments because the classification standard often varies with different backgrounds, and its establishment is relatively complicated. Some corresponding robust or non-parametric methods were proposed to handle these problems [2]; these conventional approaches are impossible to use for finding out the inherent characteristics of highdimensional non-normal data and are far from meeting the needs of analysis. They may 
lead to the problem of the "curse of dimensionality" [3,4]. For this purpose, intelligent algorithms, including the neural network method [5] and ant colony algorithm, were proposed to improve accuracy. However, they still have limitations in revealing the internal nonlinear relationship between evaluation indicators and results and have the disadvantage of local convergence [6]. Therefore, to explore the structure or features of high-dimensional data directly from the perspective of data, Friedman and Turkey [7] proposed the projection pursuit method based on exploratory data analysis (EDA) without artificial assumptions. The projection pursuit (PP) evaluation method overcomes the defect of the "curse of dimensionality" in CDA by dimension reduction and can automatically find out the internal laws of data in order to depict nonlinear characteristics of high-dimensional problems $[1,8,9]$. Therefore, the PP evaluation method without the evaluation standard has natural advantages in analyzing the issue of small-size samples.

On the other hand, it is hard to pursue a projection direction vector (PDV) for the PP index function using traditional gradient or polynomial optimization methods. Some evolutionary algorithms and intelligent swarm methods were introduced to handle this problem and have achieved specific results, but they rarely consider the multiple uncertainties of evaluation indicators. The main techniques include the differential evolution method [10], particle swarm optimization method [11], shuffled frog leaping algorithm [12], moth-flame optimization method [13], grey wolf optimization method [14], real coding based accelerating genetic algorithm (RAGA) [15] and a whale optimization strategy based on the Gaussian cloud model [16]. Nevertheless, the fruit fly optimization algorithm (FOA) [17] raised recently provides a new way to find the best PDV for the PP evaluation method, since it can conduct global information exchange and local deep search.

The FOA has the advantages of being easy to understand, simple implementation, and fewer parameters. Still, the basic FOA algorithm also has defects, such as premature convergence when the optimal solution is far from the origin, no ability to solve adverse problems, and the unstable result of the optimization [18]. The flight strategy, the search radius, and the candidate solution generation mechanism of basic FOA have been explored to overcome these shortcomings. Pan et al. [19] proposed the improved FOA (IFFO) algorithm with a better convergence rate to simulate the foraging randomness of fruit flies to some extent. Yuan et al. [20] presented the multi-swarm fruit fly optimization algorithm (MFOA) to avoid being trapped in local premature convergence and enhance the diversity of the algorithm.

On the other hand, although these improved FOAs enhance the local searching capability, they do not consider the fuzziness and stability of individual osphresis and vision behaviors in drosophila. However, the osphresis property in the foraging behavior of the fruit fly tends to characterize randomness and fuzziness. Therefore, Wu et al. [21] tried to describe randomness and fuzziness using a normal cloud model-based fruit fly optimization algorithm (CMFOA). Still, the CMFOA ignores the interval and asymmetric characteristics of the random and fuzzy uncertainties and the diversity of candidate solutions, which may not be conducive to finding the global solution. Luckily, the connection cloud model (CCM) can provide a new concept for describing randomness and fuzziness in a finite interval $[22,23]$. Like other heuristic approaches, the initial parameter setting also has pronounced effects on FOA performance [24], and the initial swarm location contributes to faster convergence [25]. In addition, the outcome performance of selecting key features is limited in conventional FOA [26]. Namely, the outcome of FOA based on chaotic location is better than those of normal distribution or uniform distribution when handling complex nonlinear problems. Consequently, to provide a reliable, optimal PDV for the PP evaluation method, an improved FOA based on the CCM and set pair analysis is addressed here to describe the randomness and fuzziness of PDV in the finite intervals.

The PP evaluation method generally projects high-dimensional data onto the onedimensional space to represent features based on a projection index function. The critical problem is to pursue the PDV that best reflects the structural characteristics of highdimensional data. It is challenging to find the optimal PDV of nonlinear problems using the 
conventional analysis methods from the above analysis. For this reason, some intelligent algorithms were introduced to handle such issues. Still, these algorithms, with many parameters and complex program code, generally have the defects of premature convergence and ignore the interaction of multiple uncertainties. In addition, the assignment of PDV inevitably involves the description of various uncertainties of the individual foraging process. As such, for high-dimensional problems, it is necessary to introduce the FOA of group collaboration and information sharing features into the PP evaluation method to optimize the PDV. However, reports on the FOA rarely focus on the description of the fuzziness and randomness of the PDV. Although Shao and Xin [27] introduced the PP method coupled with the normal cloud model to evaluate the safety of the earth-rock dam, it does not apply a normal cloud model to optimize the PDV. Nevertheless, the normal cloud model is helpful for expressing fuzzy and random characteristics in the infinite interval [16]. It is difficult to accurately describe the fuzziness of the index values on the bounds and the interval-valued measured data. Hence, there is a great demand for developing an improved FOA based on the CCM and SPA to enhance the performance of the PP evaluation method for high-dimensional problems under multiple uncertain environments.

Given the nonlinear characteristics of the slope stability evaluation under multiple uncertainties, a novel PP evaluation method using an enhanced fruit fly optimization algorithm is introduced to assess slope stability. To better achieve the global and local convergence rates and accuracy of the PP method, a new FOA based on the connection cloud model and chaotic swarm location is first presented to pursue the optimal PDV. Meanwhile, the generation mechanism of a new PDV based on the set pair analysis is adopted to improve local searchability. Namely, the identity-discrepancy-contrary (IDC) rule of set pair analysis is discussed to screen the candidate PDV, and the logistic map is also adopted to improve the mutation of the candidate PDV for the global search. These promote the algorithm to attain the goal of the candidate solution mechanism "picks the best of the best", and make the global optimization process more streamlined. The validity and feasibility of the proposed PP evaluation model were further confirmed by case study and comparative analysis with other methods. The improved FOA presented here is a balanced algorithm of the global searching capability and local acceptable optimization efficiency. It will be helpful to improve the generation mechanism and search rate of the optimal PDV, and enhance the global and local searching performance of the PP evaluation method. A quicker osphresis foraging process offers an opportunity to improve the local optimization capability and effectively depict the random and fuzzy uncertainties of individual search performance and decision.

\section{Methodology}

\subsection{Projection Pursuit}

The projection pursuit (PP) method proposed by Friedman and Turkey [7] is a powerful tool for dealing with non-normal and high-dimensional data. Its basic idea is to project high-dimensional data into a low-dimensional space concerning the objective projection function [28], then analyze the structural characteristics of original high-dimensional data with obtained projection scores to scale the possibility of a specific structure [8]. The PP evaluation method has apparent advantages in overcoming the defect "curse of dimensionality" and solving problems such as small samples and high-dimensional data, and finding out the optimal PDV that determines the accuracy of the PP evaluation method. At present, a linear projection index function is widely used in the PP method. Its corresponding model is:

$$
\begin{gathered}
Q(\boldsymbol{\alpha})=S(\boldsymbol{\alpha}) \cdot D(\boldsymbol{\alpha}) \\
S(\boldsymbol{\alpha})=\left[\frac{1}{n-1} \sum_{i=1}^{n}\left(Z_{i}-\bar{Z}\right)^{2}\right]^{0.5}
\end{gathered}
$$




$$
\begin{gathered}
D(\boldsymbol{\alpha})=\sum_{i=1}^{n} \sum_{j=1}^{n}\left(R-r_{i j}\right) \cdot I \\
R=\delta S(\boldsymbol{\alpha}) \\
r_{i j}=\left|Z_{i}-Z_{j}\right| \\
I=\left\{\begin{array}{l}
1 \text { when } R \geq r_{i j} \\
0 \text { when } R<r_{i j}
\end{array}\right. \\
Z_{i}=\sum_{p=1}^{m} \alpha_{p} x_{i p}^{*}
\end{gathered}
$$

where $Q(\alpha)$ is the projection index function. $S(\alpha)$ denotes the data dispersion characteristics of projection scores $Z(i)$ obtained based on the PDV $\alpha . D(\alpha)$ is the local density of lowdimensional data points. $\bar{Z}$ is the mean value of projection scores. $R$ represents the window radius of the local density. $I$ is the unit leap function. $r_{i j}$ is the distance.

According to the above formula, the reasonable determination of the projection direction is at the core of the PP method. However, it is difficult to determine the optimal PDV using traditional optimization methods when dealing with problems with complex topology. Consequently, the key to the successful application of the PP evaluation method lies in determining the PDV. Therefore, the CCM and chaos theory were incorporated into the FOA algorithm to depict the randomness and fuzziness of the individual PDV over finite intervals.

\subsection{FOA}

The basic FOA proposed by Pan [17] is a novel swarm intelligence optimization algorithm built on the foraging behavior of the fruit fly swarm. The detailed optimization process of the original FOA can be found in reference [17]. Based on the search strategy of population collaboration and information sharing, the basic FOA of strong robustness and reasonable convergence rate can quickly discover the optimal solution of optimization problems through the osphresis and vision foraging phases. It holds the characteristics of easy implementation and understanding of the algorithm code and has now been widely used in various fields [29-31].

The conventional FOA usually uses a fixed search step to find the optimal individual, and the greedy iterative algorithm follows. The greedy iterative algorithm seeks the global optimum near the present optimal individual in the vision foraging phase. These defects may lead to the local optima problem and inhibit the global optimization capability of the algorithm. Some scholars have presented the corresponding improved algorithms, including MSFOA [20] and IFFO [19], to enhance the global optimization capability based on the search step, candidate solution mechanism, and flight strategy of drosophila individuals. Still, most improved algorithms cannot reflect the randomness and fuzziness of individual foraging behavior and smell concentration parameters. However, the actual flying direction and distance of different drosophila individuals are random and fuzzy. The foraging behavior of individuals is different from each other when flying compared to the optimal individual. Such deviations can lead to fuzziness being inherent in the real-world optimization problems, neglect of which can cause the solutions of the issues to deviate significantly from their actual situation [32]. Thus, the MSFOA and IFFO may inevitably affect the capability of showing the structure of high-dimensional problems. The CMFOA [21] can reflect the fuzziness and randomness of the updated foraging location of individual fruit fly by a normal cloud generator instead of the original uniform random distribution in the smell search stage. Still, the normal cloud model cannot accurately describe the fuzziness at the bounds and the finite interval characteristics of the searching range. Herein, the CCM is therefore introduced here to improve the performance of the FOA. 


\subsection{Connection Cloud Model}

As is clear from the above discussion, in the traditional FOA, flavor determination calculation is based on the reciprocal of the distance, which may cause the fitness function to be unable to analyze the case where the independent variable takes a negative value or 0 . In addition, the traditional FOA may be inclined to precocious convergence for the optimal solution away from the original position because the concentration determination value away from the initial post is minimal. It can be observed that the conventional FOA easily falls into early maturity convergence. Some improved FOA methods [21] have been proposed to address these defects and have been shown to overcome these defects partly. These improved FOAs have enhanced the simulation of the foraging randomness of fruit flies and have avoided being trapped in local premature convergence to some extent. However, they cannot reflect the fuzzy characteristic of individual foraging behavior and the evaluation indicator, which may decrease the search efficiency and the ability to express data structures with uncertainties. In addition, they ignore the interval characteristic of the random and fuzzy uncertainties, and cannot accurately describe the fuzziness at the bounds [32]. The actual search direction and distance of individual foraging processes are random and fuzzy in the finite interval when real fly individuals fly to the next possible food source after learning from the optimal individual. Namely, the determination of the optimal PDV using the FOA algorithm and previous improved FOAs cannot simultaneously describe a particular projection direction's ambiguous and arbitrary characteristics. To overcome this defect, here a novel asymmetric connection cloud model (CCM) is presented to represent the actual distribution characteristic of the indicator. The CCM put forward by Wang and Jin [26] incorporated the connection numbers into the cloud model. The CCM is a powerful tool for transforming a qualitative concept into a quantitative value in a finite interval $[21,27]$. It can depict the changing tendency of the bounds and the certainty-uncertainty relationship from identity, discrepancy, and contrary elements. The CCM is presented as follows:

Denote $\mathbf{C}$ by a qualitative concept in quantitative domain $X$ with a precise value in a finite interval. If the numerical value $x \in X$ is a random realization of concept $\mathrm{C}$, then the quantitative description for the certainty-uncertainty relationship between $x$ and the concept $\mathbf{C}$ is:

$$
\mu=\left[1-\left(\frac{x-E x}{y}\right)^{2}\right]^{k}
$$

where $\mu \in[0,1]$ is the connection degree. $y$ and $x$ satisfy the normal distribution $N\left(E n, H e^{2}\right)$ and $N\left(E x, y^{2}\right)$, respectively. $E x, E n$, and He denote expectation, entropy, and hyper entropy, respectively; $y$ represents the left or right branch of cloud width; $k$ is the order of the distribution density function. These parameters of $E x, E n, H e, y$, and $k$ are collectively named as the numerical characteristics of the CCM. The numerical characteristics are given as:

$$
\begin{gathered}
E x=\frac{L_{\max }+L_{\min }}{2} \\
E n=\frac{\alpha}{\sqrt{2 k+3}} \\
k=\frac{\lg 0.5}{\lg \left[1-\left(\frac{l-E x}{\alpha}\right)^{2}\right]} \\
\alpha^{\prime}=y \sqrt{2 k+3}
\end{gathered}
$$

where $\alpha^{\prime}$ is the modified width of the left or right branch of cloud; $l$ denotes the indicator value responding to the connection degree of 0.5 . 


\section{Development of the PP Evaluation Method Using Improved FOA}

\subsection{Basic Principle}

The basic principle of the PP evaluation method using the improved FOA based on CCM and SPA is presented as follows: Firstly, initialize the parameters and normalize measured data. Next, construct the PP index function and the optimization model. Then, combining the measured data, find the optimal PDV most likely to represent the original high-dimensional data structure or feature using the improved FOA. Namely, the digital characteristic parameters of CCM, Ex, and En are adopted to express the randomness and fuzziness of the optimal food source location and search range in the smell search stage, and the correlation between random and fuzzy characteristics and smell concentration parameters of the individual search. At the same time, based on the rule of identitydiscrepancy-contrary (IDC) of set pair analysis [16,28], the candidate mechanism of the best projection direction is enhanced by the greedy strategy, and an adaptive entropy accelerates the local convergence speed with the specific number of iterations. Then next, the mutation search radius near the location of the primary optimal drosophila is produced by the chaos theory to increase the diversity and ambiguity of individuals to prevent the algorithm from falling into the local optimum and ensure the obtaining of the solution of the optimal projection direction. Finally, high-dimensional measured data are projected into a one-dimensional subspace to investigate the structure based on the optimal PDV found.

\subsection{Evaluation Procedure}

The detailed evaluation procedure using the PP evaluation method based on the improved FOA consists of six steps, as illustrated in Figure 1.

Step 1: Standardize indicator values of samples. As we know, different evaluation indexes have various dimensions and no uniform range, so the measured values of indexes should be normalized to reduce their impacts. Responding to the benefit indicator that the greater the attribute value the better the indicator is, the standardized model is:

$$
x_{i p}^{*}=\left(x_{i p}-x_{\min }^{p}\right) /\left(x_{\max }^{p}-x_{\min }^{p}\right)
$$

where $x_{i p}^{*}$ and $x_{i p}$ are the normalized value and the measured amount of indicator $p$ of the $i$ th sample, respectively. $x_{\max }^{p}$ and $x_{\min }^{p}$ denote the maximal and minimal amounts of measured indicator $p$, respectively. While for the cost indicator that the smaller the attribute value the better the index is, the corresponding model is given as:

$$
x_{i p}^{*}=\left(x_{\max }^{p}-x_{i p}\right) /\left(x_{\max }^{p}-x_{\min }^{p}\right)
$$

Step 2: Initialize the PDV and parameters of the improved FOA.

$$
X_{i j}=L B_{j}+\left(U B_{j}-L B_{j}\right) \cdot \operatorname{rand}() / 2
$$

$X_{i j}$ denotes the initial PDV; $L B_{j}$ and $U B_{j}$ are the lower and upper bounds of the $j$ th evaluation indicator; rand() is a function to produce a random number obeying the uniform distribution on the interval $[0,1]$.

Step 3: Construct the projection index function. The core of the PP evaluation method is to find an optimal PDV based on the projection index function to characterize the structural features of high-dimensional data. Here, a linear projection index function $Q(\alpha)$ is adopted. The corresponding pseudo-code for the swarm projection pursuit algorithm is shown in Algorithm 1.

Step 4: Identify the optimization model of the PDV. For the given samples, the projection index function $Q(\alpha)$ only varies with the PDV of $\alpha$, so different PDVs reflect various structural features, among which, the optimal PDV can best reveal the structural characteristics of high-dimensional data. Therefore, the optimal PDV can be found out by maximizing the projection index function. The corresponding optimization model is: 


$$
\left\{\begin{array}{c}
\max [Q(\boldsymbol{\alpha})] \\
\text { s.t. } \sum_{p=1}^{m} \alpha_{p}^{2}=1
\end{array}\right.
$$

Step 5: Find out the optimal PDV based on the improved FOA. According to the analysis as mentioned above, the foraging process of the fruit fly population is to update the search position dynamically based on the perceived smell concentration. Still, the location of the fruit fly population is randomly distributed in the area. The judgment of smell concentration is also fuzzy for each fruit fly, so the individual search range and direction are randomness and fuzziness. Moreover, the high-dimensional data themselves contain random and ambiguous indicators. However, the basic FOA and conventional improved FOAs seldom consider multiple uncertainties in the finite interval. Therefore, it is hard to apply them to find the optimal PDV of such problems. As such, the numerical characteristics of the CCM were attempted here to outline the randomness, fuzziness, and stability of the optimal PDV during the optimization process of the PDV using the new, improved FOA.

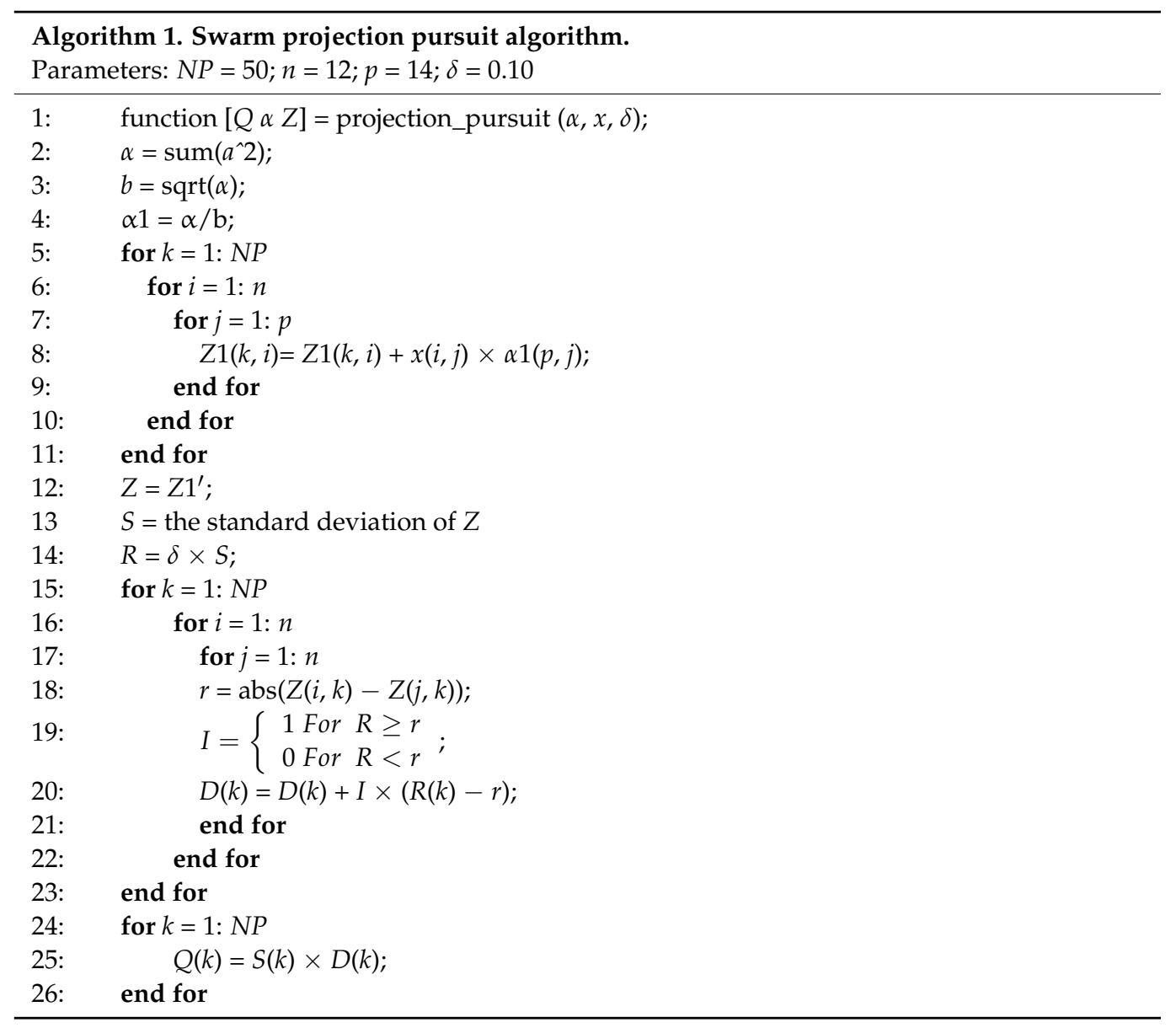

In the novel improved FOA proposed here, the PDV obtained from the latest optimization is characterized by the expectation $E x$ of the CCM, and the random number $y$ generated is used to depict the individual search radius of random and fuzzy characteristics. The entropy $E n$ is adjusted adaptively and dynamically with the iteration number to enhance the local search convergence rate. At the same time, the theory of set pair analysis is introduced here to screen the updating of individual PDV near the optimal PDV to improve the candidate solution mechanism. Firstly, it substitutes the candidate PDV into Equation (8) to calculate the connection degree. Then, depending upon the obtained connection degree, the candidate PDV is further analyzed by the rule of identity-discrepancy-contrary (IDC). Herein, the corresponding identity and contrary relationships are defined as follows: 
There is an identity relationship between the individual PDV produced randomly and the optimal PDV of swarm obtained by the latest iteration when the connection degree is more significant than 0.5 . At the same time, there is a contrary relationship when the gained connection degree is less than 0.5 . The individual PDV with an identity relationship meets the candidate requirements and can enter the optimization calculation of the PDV. In contrast, the candidate PDV with a contrary relationship needs to be regenerated before the measure. The corresponding model is written as:

$$
\begin{gathered}
k=\left\{\begin{array}{r}
k+1 \text { For the connection degree } \mu \geq 0.5 \\
k \text { otherwise }
\end{array}\right. \\
\text { En }=\operatorname{rand}() \cdot \frac{\left(U B_{j}-L B_{j}\right)}{2} \cdot \exp \left[\ln \left(\frac{B \text { eta } a_{\text {lower }}}{\text { Beta }_{\text {upper }}}\right) \cdot\left(\frac{g}{\text { maxgen }}\right)\right]
\end{gathered}
$$

where $k$ is the $k$ th PDV. Obviously, in the smell searching phase of the improved FOA, the candidate solution generation mechanism of the PDV based on the set pair analysis is improved relative to the basic FOA and other improved FOAs. It can promote faster aggregation to the optimal PDV obtained by the latest iteration and enhance the convergence performance of the FOA algorithm. Meanwhile, the improved FOA proposed here uses chaos theory to produce the new PDV near the optimal PDV. These advantages will recover from the defect of the basic FOA fallof easily falling into local convergence and improve the diversity of the candidate PDV. The corresponding algorithm is given as:

$$
\alpha(g+1)=\eta \cdot \alpha(g)[1-\alpha(g)]
$$

where $g$ is the generation number; $\eta$ denotes the coefficient of chaos. Thus, the improved FOA expands the diversity of the PDV through the chaotic mechanism to increase the variety of candidate solutions, which can prevent the optimization process from falling into the problem of local optima.

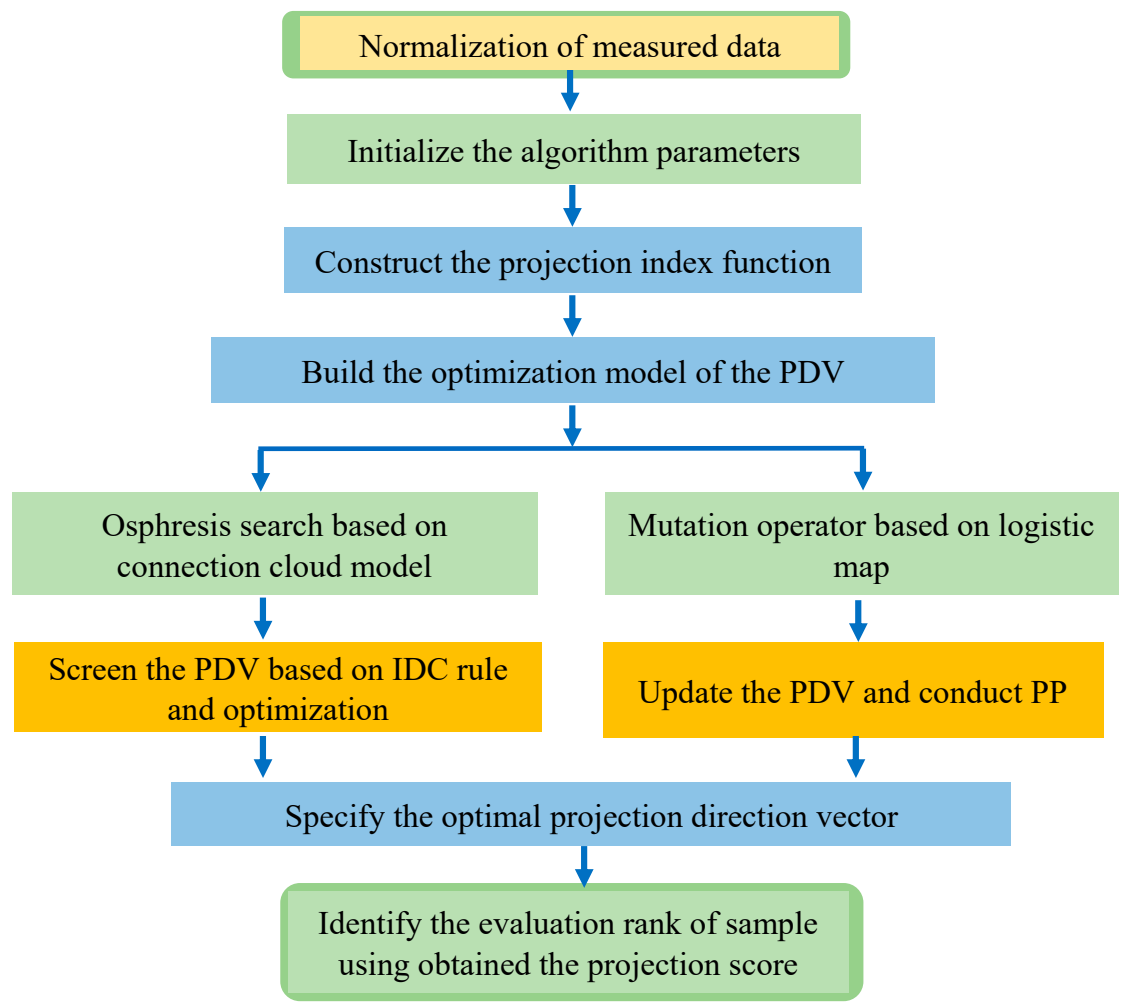

Figure 1. Schematic diagram of the evaluation procedures by the new PP evaluation method based on the improved FOA. 
In general, the improved FOA can reflect the randomness and fuzziness of the individual judgment and decision of smell concentration, reflect the randomness and fuzziness of independent review and smell concentration and balance the global search capability and local optimization capability. Solving PDV using the improved FOA includes four phases: (1) Initialization of algorithm parameters and the PDV and projection parameters. (2) Generation of the PDV near the latest optimal solution using the set pair analysis and the CCM with an adaptive entropy En. (3) Expansion of the PDV diversity by chaotic mutation. (4) Iterative solution of the optimal PDV. Their pseudo-codes are illustrated as shown in Algorithm 2.

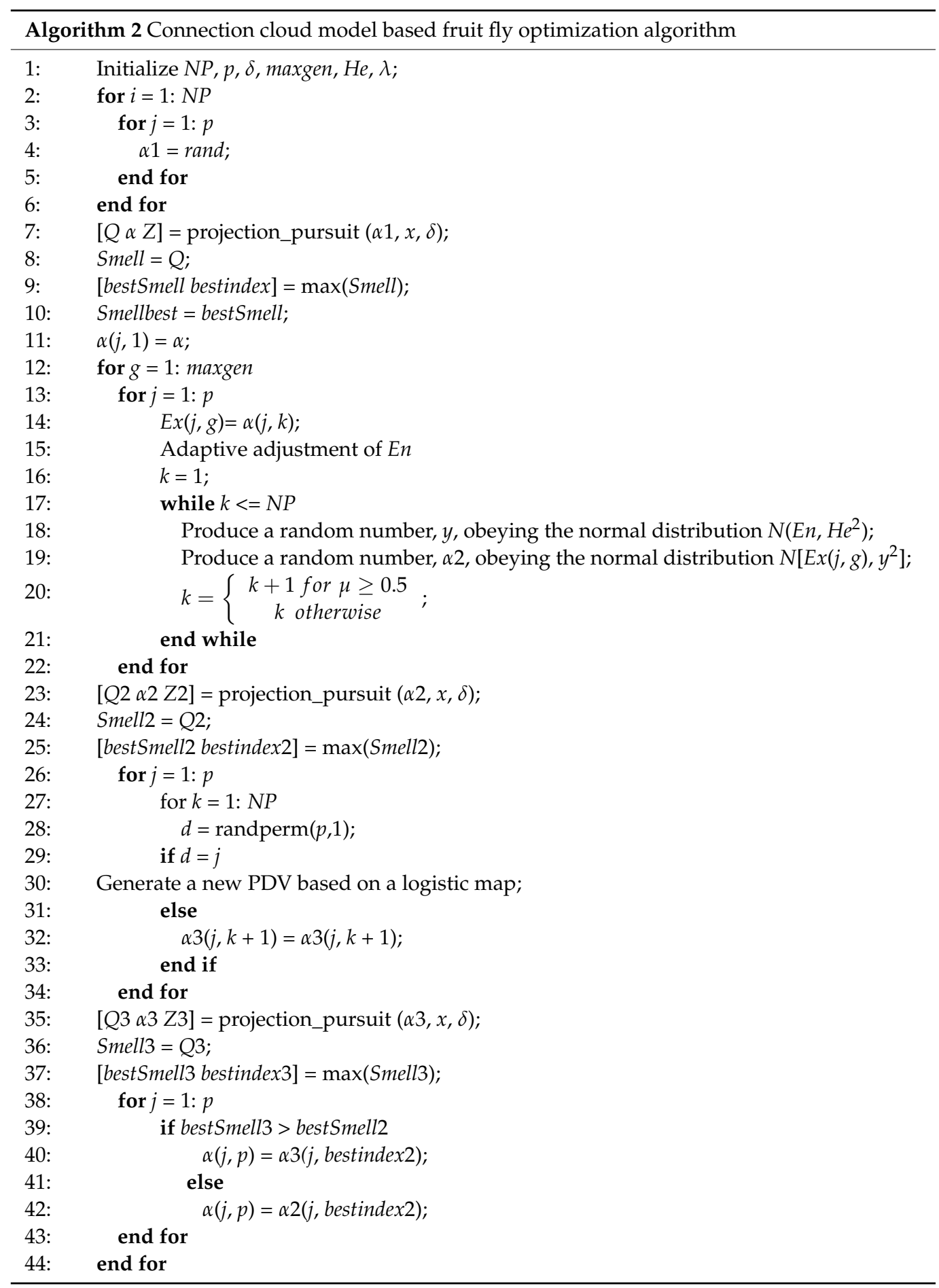


Step 6: Calculate the projection score of samples based on the obtained optimal PDV to conduct a comprehensive analysis.

\section{Case Study}

\subsection{Data}

An illustrative example of slope stability in Ref. [5] was demonstrated here to confirm the applicability and reliability of the PP evaluation method based on the novel FOA proposed here. As we know, slope stability depends upon uncertain geological information, so slope stability evaluation is complex. Evaluation methods of slope stability mainly exist through quantitative and qualitative evaluation methods. The classical quantitative evaluation method-the engineering geology analogy method of simple and easy operation advantages-is commonly used in engineering practice. Nevertheless, it is hard to account for the uncertainty of the evaluation indicators due to the lack of unified standards. Limit equilibrium methods such as the Fellenius, Bishop and Janbu methods need certain hypothetical conditions to be met, so their applications have specified limitations. To overcome these shortcomings, various quantitative analysis methods, including the fuzzy sets method, the probabilistic theory, artificial neural network (ANN) method, and the SVM, have been developed for slope stability analysis and have demonstrated successful performances. However, they cannot describe multiple uncertainties of slope stability problems [15]. Predicting unstable slopes is theoretically a process of functional approximations [33], so backpropagation neural networks are an effective evaluation method for slope stability [34]. The real coding-based accelerating genetic algorithm (RAGA), based on real number coding, improves the standard genetic algorithm [35], and an adaptive global optimization probabilistic search algorithm. It has the advantages of needing no decoding process, simple genetic operation, and easy high-precision numerical optimization. Thus, the RAGA provides a new effective way to solve the optimal projection direction for optimization problems. Artificial intelligence-based methods often cannot explicitly link evaluation parameters and rank potential and have certain inherent drawbacks.

In general, previous studies have concentrated on a single type of uncertainty and are always set up based on the existing classification standards or empirical rules. However, establishing a classification standard for slope stability is relatively complicated, so those methods may have limitations in assessing slope stability. The PP evaluation method without the evaluation standard still has disadvantages in its difficulty in determining the projection direction, despite its natural advantages in assessing the slope stability. To this end, various intelligent approaches have been undertaken to find rational PDV and beneficial progress has been made. Although these robust methods may achieve a better PDV to some extent, they rarely examine the multiple uncertainties of PDV. Recently, to express fuzzy and random characteristics, the CMFOA has been taken to find PDV, but it is difficult to accurately describe the fuzziness of the index values on the bounds. Hence, simultaneously considering the randomness and fuzziness of evaluation indicators and the PDV in the finite intervals, a compelling slope stability assessment is required, by employing efficient methods for considering multiple uncertainties.

Comparisons of results with the neural network method and PP evaluation methods based on the primary and improved FOAs and RAGA were also carried out. Slope hazard, one of the most important geological disasters, produces significant losses of social economy and human life [36]. Therefore, the evaluation of slope stability has considerable social and economic significance. However, slope instability caused by long geological action involves various uncertain factors [37], so its evaluation is a complex uncertainty problem with multi-dimensional and nonlinear data. In the case study, there were 14 evaluation indexes including the height difference $C_{1}$, slope angle $C_{2}$, the relationship between the flood level and landslide shear outlet elevation $C_{3}$, sliding body area $C_{4}$, water permeability of sliding body $C_{5}$, rainstorm intensity $C_{6}$, deformation failure sign $C_{7}$, material structure $C_{8}$, occurrence change of active surface $C_{9}$, the strength of slip zone $C_{10}$, the occurrence of shear outlet $C_{11}$, the human activity condition $C_{12}$, the composition of rock mass $C_{13}$, 
and the dip angle of rock stratum $C_{14}$. The grade of the slope stability was divided into five levels: unstable (I), relatively unstable (II), basically stable (III), relatively stable (IV), and stable (V). Normalized values of indicators for samples are shown in Table 1.

Table 1. Indicator values of the samples.

\begin{tabular}{cccccccccccccccc}
\hline \multirow{2}{*}{ Samples } & Slope Name & \multicolumn{10}{c}{ Evaluation Indicators } \\
\cline { 3 - 15 } & & $\boldsymbol{C}_{\mathbf{1}}$ & $\boldsymbol{C}_{\mathbf{2}}$ & $\boldsymbol{C}_{\mathbf{3}}$ & $\boldsymbol{C}_{\mathbf{4}}$ & $\boldsymbol{C}_{\mathbf{5}}$ & $\boldsymbol{C}_{\mathbf{6}}$ & $\boldsymbol{C}_{\mathbf{7}}$ & $\boldsymbol{C}_{\mathbf{8}}$ & $\boldsymbol{C}_{\mathbf{9}}$ & $\boldsymbol{C}_{\mathbf{1 0}}$ & $\boldsymbol{C}_{\mathbf{1 1}}$ & $\boldsymbol{C}_{\mathbf{1 2}}$ & $\boldsymbol{C}_{\mathbf{1 3}}$ & $\boldsymbol{C}_{\mathbf{1 4}}$ \\
\hline $\mathbf{1}$ & Huanglashi 1 & 0.32 & 0.79 & 0.83 & 0.82 & 1.00 & 0.76 & 0.90 & 0.90 & 1.00 & 1.00 & 0.50 & 1.00 & 0.88 & 0.10 \\
2 & Xintan & 1.00 & 0.90 & 0.13 & 0.47 & 0.87 & 1.00 & 1.00 & 0.80 & 0.77 & 0.80 & 1.00 & 0.70 & 0.80 & 0.10 \\
3 & Tianbao & 0.17 & 0.86 & 1.00 & 0.74 & 0.80 & 1.00 & 0.77 & 0.70 & 0.83 & 0.80 & 1.00 & 1.00 & 0.30 & 0.80 \\
4 & Baota & 0.52 & 0.75 & 0.63 & 0.14 & 0.86 & 1.00 & 0.83 & 0.60 & 0.47 & 0.65 & 1.00 & 0.70 & 0.35 & 0.55 \\
5 & Yunyangxicheng & 0.33 & 0.87 & 0.69 & 0.44 & 1.00 & 0.80 & 1.00 & 0.90 & 0.53 & 0.85 & 0.63 & 0.70 & 0.36 & 0.49 \\
6 & Huanglashi 2 & 0.24 & 0.86 & 0.20 & 0.92 & 0.77 & 0.70 & 0.63 & 0.10 & 0.93 & 1.00 & 0.50 & 0.70 & 0.88 & 0.10 \\
7 & Liulaiguan & 0.48 & 0.90 & 0.72 & 0.59 & 0.67 & 0.20 & 0.70 & 0.50 & 0.57 & 1.00 & 0.85 & 0.60 & 0.88 & 0.10 \\
8 & Baiyian & 0.38 & 0.80 & 0.66 & 0.45 & 0.60 & 0.30 & 0.90 & 0.70 & 0.45 & 0.25 & 0.65 & 0.30 & 0.70 & 0.90 \\
9 & Xiangjiawan & 1.00 & 0.80 & 0.96 & 0.41 & 0.47 & 0.30 & 0.43 & 0.30 & 0.87 & 0.50 & 0.80 & 0.30 & 0.50 & 0.20 \\
10 & Baihuanping & 0.44 & 0.45 & 0.69 & 0.10 & 0.40 & 0.30 & 0.57 & 0.20 & 0.50 & 0.80 & 0.40 & 0.30 & 0.87 & 0.50 \\
11 & Fanjiaping & 0.48 & 0.56 & 0.66 & 0.14 & 0.47 & 0.20 & 0.57 & 0.20 & 0.57 & 0.70 & 0.28 & 0.30 & 0.30 & 0.70 \\
12 & Jiuxianping & 0.33 & 0.49 & 0.67 & 0.27 & 0.57 & 0.80 & 0.43 & 0.10 & 0.47 & 0.40 & 0.20 & 0.20 & 0.27 & 0.30 \\
\hline
\end{tabular}

\subsection{Model Implementation}

The corresponding Matlab program was coded based on the above-discussed evaluation procedures in Section 3. Based on the measured values of sample, the optimal PDV is obtained as $\alpha=(0.094,0.443,0.122,0.307,0.276,0.232,0.278,0.261,0.248,0.261,0.466,0.244$, $0.063,0.023)$, using the improved FOA with the population size of 50 , the iteration number of 100 , the adaptive control coefficient $\zeta$ of 7 , and the corresponding maximal value of the projection index function $Q(\alpha)$ is 1.743. The minimal and maximal values of the Beta are $10^{-5}$ and 0.5 , respectively. Projection scores for samples obtained by real-coding-based accelerating genetic algorithm (RAGA), IFOA, CFOA, and improved FOA proposed here are illustrated in Figure 2 and Table 2. Their classification results are given in Table 3.

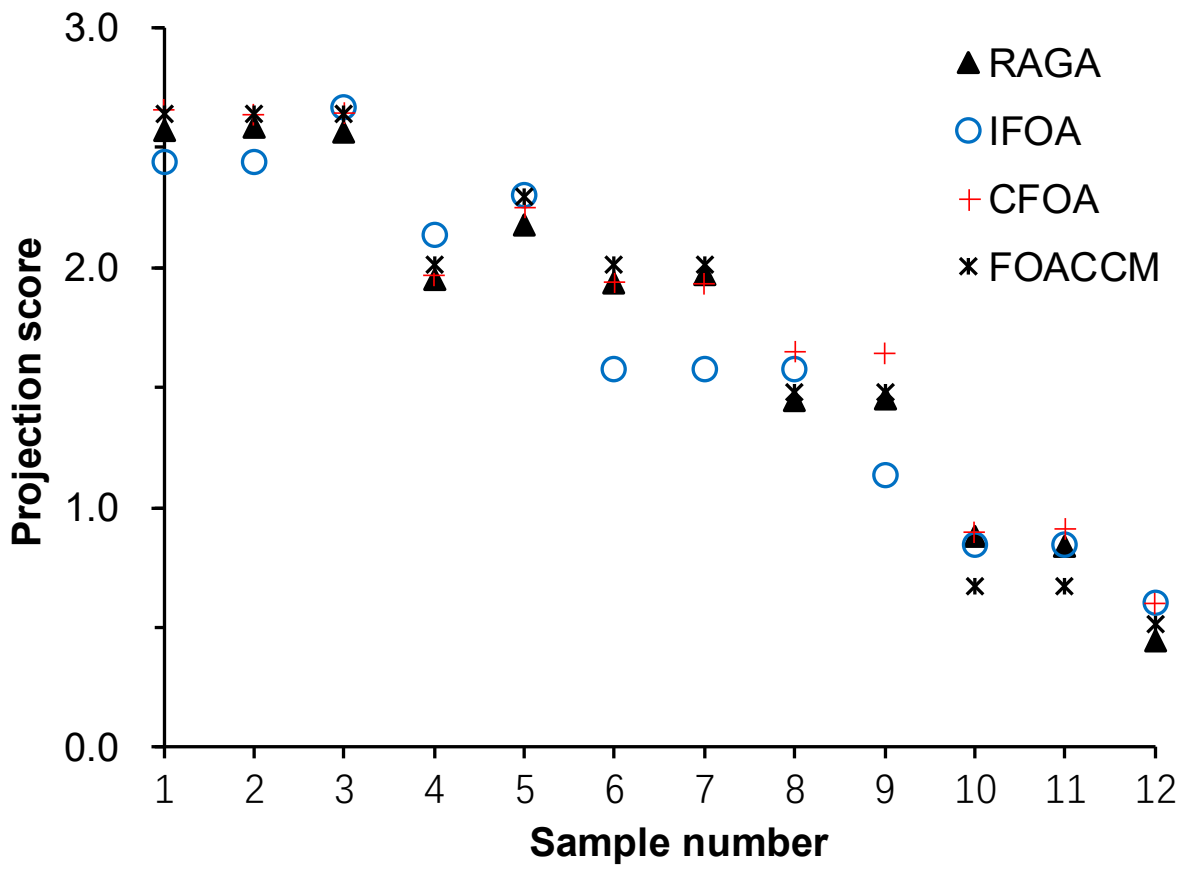

Figure 2. Results of projection scores of samples. 
Table 2. Projection scores of samples based on the optimal PDVs from different algorithms.

\begin{tabular}{ccccccccccccc}
\hline Samples & $\mathbf{1}$ & $\mathbf{2}$ & $\mathbf{3}$ & $\mathbf{4}$ & $\mathbf{5}$ & $\mathbf{6}$ & $\mathbf{7}$ & $\mathbf{8}$ & $\mathbf{9}$ & $\mathbf{1 0}$ & $\mathbf{1 1}$ & $\mathbf{1 2}$ \\
\hline RAGA [38] & 2.570 & 2.583 & 2.562 & 1.949 & 2.179 & 1.934 & 1.970 & 1.443 & 1.451 & 0.878 & 0.836 & 0.445 \\
IFOA & 2.440 & 2.440 & 2.668 & 2.138 & 2.302 & 1.574 & 1.574 & 1.574 & 1.138 & 0.848 & 0.848 & 0.602 \\
CFOA & 2.656 & 2.637 & 2.640 & 1.964 & 2.248 & 1.942 & 1.930 & 1.646 & 1.641 & 0.896 & 0.908 & 0.601 \\
CCMFOA & 2.639 & 2.638 & 2.639 & 2.009 & 2.294 & 2.009 & 2.009 & 1.480 & 1.480 & 0.674 & 0.674 & 0.516 \\
\hline
\end{tabular}

Table 3. Comparison of results from different methods.

\begin{tabular}{ccccccccccccc}
\hline Samples & $\mathbf{1}$ & $\mathbf{2}$ & $\mathbf{3}$ & $\mathbf{4}$ & $\mathbf{5}$ & $\mathbf{6}$ & $\mathbf{7}$ & $\mathbf{8}$ & $\mathbf{9}$ & $\mathbf{1 0}$ & $\mathbf{1 1}$ & $\mathbf{1 2}$ \\
\hline ANN [5] & I & I & I & II & II & III & III & IV & IV & V & V & V \\
RAGA [38] & I & I & I & III & II & III & III & IV & IV & V & V & V \\
IFOA & I & I & I & II & II & III & III & III & IV & V & V & V \\
CFOA & I & I & I & III & II & III & III & IV & IV & V & V & V \\
CCMFOA & I & I & I & III & II & III & III & IV & IV & V & V & V \\
\hline
\end{tabular}

\subsection{Results Analysis}

The projection score indicates the varying level of the slope. Their orders from large to small for the projection scores obtained by the improved FOA were samples 3, 1, 2, 5, $4,6,7,9,8,10,11$, and 12 . The grade of samples 1,2 , and 3 was unstable I; that of sample 5 was relatively unstable II; those of samples 4,6 , and 7 were stable III; those of samples 8 and 9 can be rated as relatively stable IV; and samples 10,11, and 12 can be ordered as stable $\mathrm{V}$, respectively. The evaluation results were broadly consistent with the results of the neural network method, except sample 4 . The result indicates that the proposed method is practical and feasible. At the same time, it is seen that the evaluation results from the PP evaluation method can directly depict the data from multiple variables and the degree of slope stability with one-dimensional variables.

In contrast, the neural network method has a black box effect and cannot reflect the direct relationship between the evaluation indicators and the evaluation results. Moreover, the optimal PDV changed with different optimization methods despite giving the same classification to a specific sample. This may suggest that the optimization of the projection direction is too complicated to solve based only on the projection index function for the problem under multiple uncertain environments.

\subsection{Comparison and Discussions}

The values of the projection index function $Q(\alpha)$ versus iteration numbers obtained from different algorithms are presented in Figure 3, and the corresponding optimal PDVs are listed in Table 4 and Figure 4. It was observed in Figure 3 that the absolute value of the projection index function obtained from the improved FOA was the largest, and about $25 \%$ and $13 \%$ higher than those of CFOA and IFOA under the same initial PDV. These results may indicate that the generation mechanism of the candidate PDV in the basic FOA may lead to a local optimum, while the improved FOA using chaos theory can enhance the global searching performance of the PP evaluation method. The generation mechanism of candidate PDV based on the connection cloud model and IDC analysis is beneficial for improving the search rate of the optimal PDV. Meanwhile, the evaluation results from the improved FOA were easier for determining the state of the slope stability and more convenient for application relative to other methods. It was also observed that the number of computational iterations for obtaining the optimal PDV depending on improved FOA, the IFOA, and the CFOA were 39, 19, and 61, respectively. The IFOA reached the optimal solution fastest. The search rate was about 2.2 and 1.1 times faster than those of the CFOA and improved FOA, respectively, but the projection index function value was approximately $30 \%$ lower than that of the improved FOA, while the search rate and the projection index function value of the enhanced FOA were about 0.7 times and $42 \%$ higher than those of the CFOA. 
Overall, although the IFOA has good global search efficiency, since it pursues the optimal solution faster than the improved FOA and the CFOA, the capability of the local search was worse. The enhanced FOA is a swarm intelligence algorithm with global and local abilities in harmony. It can conduct deep mining and local refinement of the convergence region and concurrently comprehensively optimizes the space outside the convergence region using the chaotic mutation operator. Hence, the improved FOA proposed here can ensure the search efficiency and precision of the optimal PDV and effectively describe the randomness and fuzziness of the candidate optimal PDV in a finite interval.

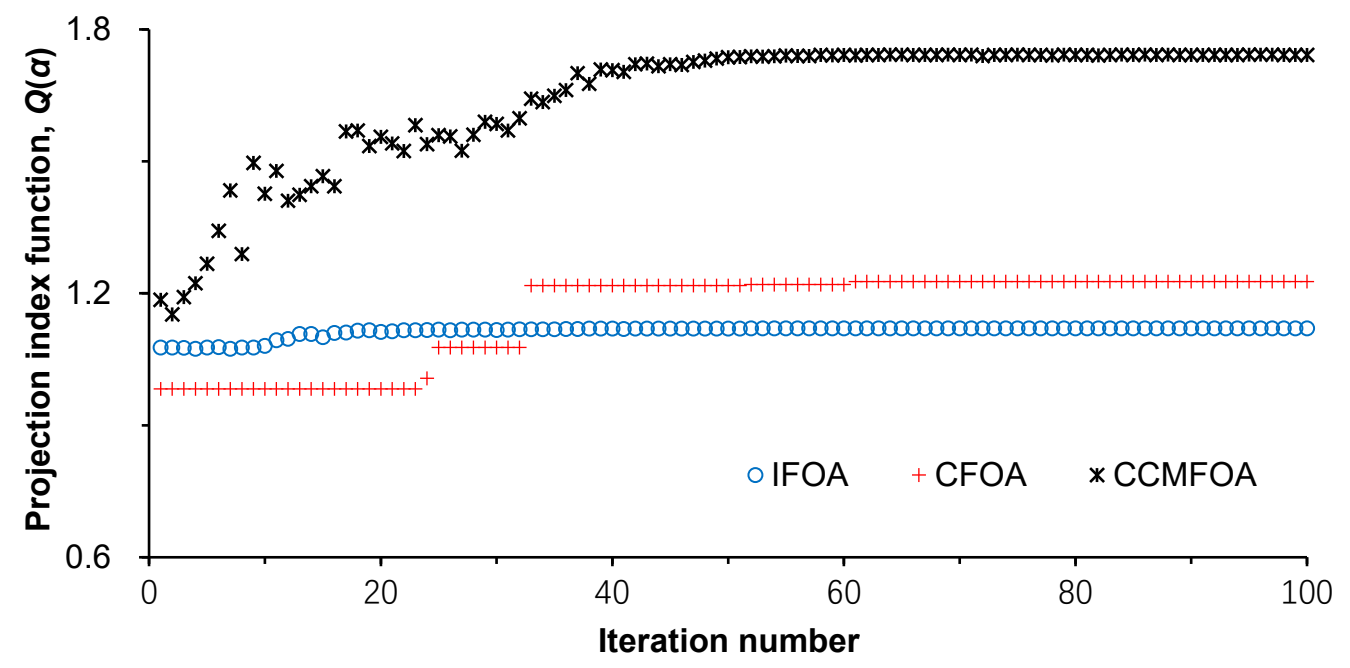

Figure 3. Optimization process along iteration number.

Table 4. Comparison of the optimal PDV.

\begin{tabular}{|c|c|c|c|c|c|c|c|c|c|c|c|c|c|c|}
\hline $\begin{array}{c}\text { Optimization } \\
\text { Algorithm }\end{array}$ & 1 & 2 & 3 & 4 & 5 & 6 & 7 & 8 & 9 & 10 & 11 & 12 & 13 & 14 \\
\hline RAGA [38] & 0.336 & 0.535 & 0.193 & 0.389 & 0.153 & 0.291 & 0.716 & 0.338 & 0.502 & 0.667 & 0.733 & 0.666 & 0.144 & 0.215 \\
\hline IFOA & 0.106 & 0.141 & 0.134 & 0.106 & 0.164 & 0.374 & 0.442 & 0.327 & 0.217 & 0.236 & 0.346 & 0.371 & 0.042 & 0.328 \\
\hline CFOA & 0.297 & 0.317 & 0.146 & 0.333 & 0.209 & 0.224 & 0.240 & 0.343 & 0.340 & 0.271 & 0.325 & 0.197 & 0.117 & 0.251 \\
\hline CCMFOA & 0.094 & 0.443 & 0.122 & 0.307 & 0.276 & 0.232 & 0.278 & 0.261 & 0.248 & 0.261 & 0.466 & 0.244 & 0.063 & 0.023 \\
\hline
\end{tabular}

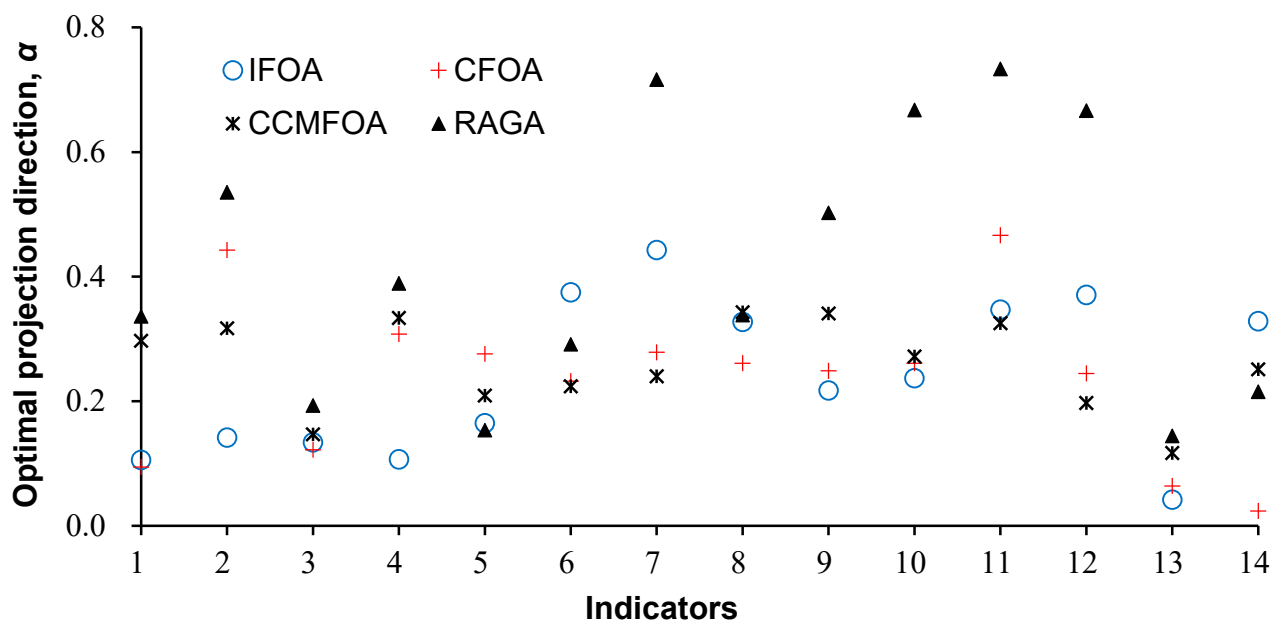

Figure 4. Optimal projection direction of the indicator.

The evaluation of slope stability is a complicated problem with various uncertainty factors. The case study shows that the improved FOA can overcome the shortcomings of the basic FOA in the solution of PDV and has the advantages of better efficiency and accuracy. The proposed algorithm can significantly enhance global and local search capabilities. After 
all, it takes advantage of randomness, fuzziness, and traversal characteristics. It has the following benefits over other methods:

(1) Compared with the neural network evaluation method, the proposed method overcomes the burden of knowledge acquisition based on many samples. And it also can directly obtain the relationship between the evaluation indicators and the classification results, while the classification results obtained from the neural network method are from the black box effect.

(2) The improved FOA can apply expectation $E x$ to memorize a possible optimal PDV. The search range and learning degree of the candidate PDV are also characterized intelligently by the entropy $E n$. The larger the $E n$, the larger the individual fluctuation range is. The hyper entropy can further depict the stability of individual learning and demonstrate the power of personal understanding. These characteristics can promote the optimal PDV obtained from elite individuals. It also avoids the limitation of the normal cloud model that requires the normal distribution of optional solution parameters.

(3) The generation mechanism of the new PDV is strengthened by the set pair analysis. Namely, the identity and contrary relationships between the candidate PDV and the optimal PDV obtained from the latest optimization are analyzed according to the connection degree. The candidate PDV generated randomly is screened before the complex calculation of PP. It enables the algorithm to achieve the goal of the candidate solution mechanism "picks the best of the best" and makes the global optimization process more streamlined.

(4) The candidate PDV generated near the optimal solution based on logistic mapping ensures the optimal global solution is obtained with ergodicity, randomness, and diversity. Thus, the algorithm can jump out of the local optimum while quickly reaching the global solution.

\subsection{Sensitivity Analysis}

To analyze the influence of the parameters on the present algorithm, the sensitivity analysis of the primary parameters population size and iteration number is performed here. The corresponding calculation results are shown in Figures 5 and 6.

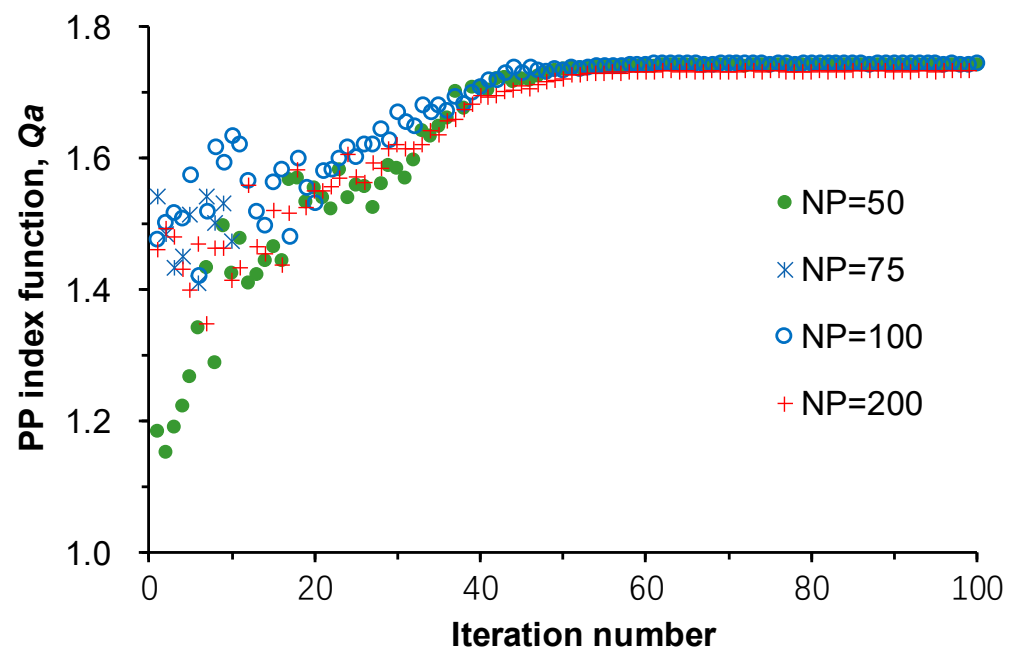

Figure 5. Sensitivity analysis of population size for the algorithm.

Figure 5 shows the results of the sensitivity analysis of the population size parameter. The results were obtained with the same initial projection direction of $\alpha=(0.111,0.146,0.140$, $0.096,0.171,0.375,0.383,0.340,0.228,0.248,0.363,0.389,0.038,0.038,0.329)$. As shown in Figure 5, the maximum projection index values of the population size parameters $N P=50,75,100$, and 200 were very close. The number of computational iterations reaching the maximum value was similar, indicating that the model is relatively insensitive to the population size parameters in the same initial projection direction and verifies the model's stability. 


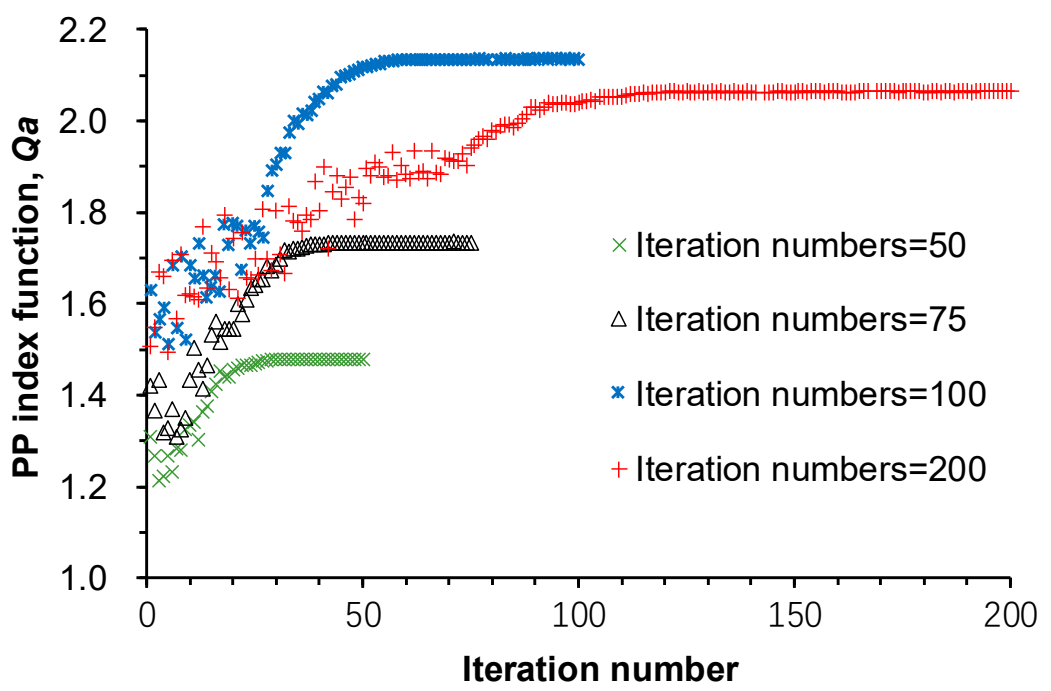

Figure 6. Sensitivity analysis of iteration numbers for the algorithm.

Figure 6 illustrates the results of the sensitivity analysis of the iteration number parameters. The result was likewise obtained based on calculations under the same initial projection direction. It was seen from Figure 6 that the maximum projection index values were affected by the iteration number parameters. With iteration numbers maxgen $=50,75,100$, the maximum projection index value increased with increasing iteration numbers. However, the projection index value of iteration numbers maxgen $=200$ was lower than iteration numbers maxgen $=100$, indicating that the increase of iteration numbers could improve the analysis effect to some extent. Therefore, the present model can achieve the target with fewer iterations, showing that the current algorithm has high computational efficiency.

\section{Conclusions}

Conventional optimization algorithms are not robust enough to deal with the uncertainties of fuzziness and randomness in the finite interval. Although the PP evaluation method is a common tool to analyze and cluster the actual engineering problems of high-dimensional data, it raises the issue of detecting the PDV to reflect the structure or characteristics of the original non-normal data. Here, a novel fruit fly optimization algorithm based on the CCM and set pair analysis to optimize the PDV and the PP method based on the improved FOA are investigated to analyze the structural characteristics of data with randomness and fuzziness. An illustrative example of slope stability further verifies the reliability and applicability of the proposed method, and principal conclusions are concluded as follows:

(1) The slope stability evaluation involves various uncertain indicators, and there is no unified evaluation standard and evaluation index system. These uncertainties restrict the application of evaluation methods relying on classification criteria for slope stability. The CCMFOA-based PP approach without the rating standard provides a refreshing concept for examining slope stability directly through small-size measured data.

(2) Case study indicates that the CCMFOA overcomes the defects of the original FOA or other improved FOAs, and can fully depict the symmetric structure and the randomness and fuzziness characteristics of the PDV. Meanwhile, the projection rate of the model presented here is faster than other algorithms. It has a high convergence accuracy relative to other improved FOAs based on the normal distribution for the problem with small-size samples under uncertain environments. Thus, the proposed PP evaluation method based on the enhanced FOA does not require the existing evaluation classification criteria and can fully explore and depict the structure and information of high-dimensional data of samples in one-dimensional space. It provides an alternative way to assess slope stability under multiple uncertainties. 
(3) Compared with the evaluation correctness and computational convergence rate of the basic FOA and conventional improved FOAs, the generation mechanism of the candidate PDV incorporated with the CCM and IDC principle of SPA can accelerate the osphresis foraging process and enables the improvement of the local fine optimization capability and to the effective depiction of the random and fuzzy uncertainties of individual search performance and decision. At the same time, adaptive control of search range and chaotic mutation increases the diversity and ergodicity of the improved FOA. The enhanced FOA proposed here is a balanced algorithm of the global searching capability and local acceptable optimization efficiency.

(4) Algorithmic sensitivity analyses of population size and the iteration number in the same initial projection direction show that population size has less impact on the present model. In contrast, iteration number has some role in the simulation results, while also verifying that the current model has good stability and computational efficiency.

(5) Although the improved FOA can provide more accurate PDV for the PP method than other FOAs, producing the excellent initial conditions for this improvement still needs further investigation. In addition, the adaptability of the proposed model still needs to be further explored through more examples and practical applications in the future.

Author Contributions: Supervision, M.W.; Methodology, M.W. and Y.W.; Writing—original draft, M.W. and Y.W.; Funding acquisition, M.W. and J.J.; Writing-review and editing, M.W., Y.W., F.S. and J.J.; Data curation, Software, M.W. and Y.W.; Validation, M.W. and Y.W. All authors have read and agreed to the published version of the manuscript.

Funding: Financial support was partially provided by the National Key Research and Development Program of China under Grant (No. 2017YFC1502405) and the National Natural Sciences Foundation of China (No. 41172274).

Institutional Review Board Statement: Not applicable.

Informed Consent Statement: Not applicable.

Data Availability Statement: Not applicable.

Acknowledgments: Financial support partially provided by the National Key Research and Development Program of China under Grant (No. 2017YFC1502405) and the National Natural Sciences Foundation of China (No. 41172274) is gratefully acknowledged. The authors also thank the reviewers for their thorough reviews and suggestions that helped to improve this paper.

Conflicts of Interest: The authors declare that there is no conflict of interest regarding the publication of this paper.

\section{References}

1. Li, Z.Y. Projection pursuit technology (PPT) and its progress of application. Chin. J. Nat. 1997, 19, 224-227.

2. Jorba, L.; Adillon, R. A generalization of trapezoidal fuzzy numbers based on modal interval theory. Symmetry 2017, 10, 198. [CrossRef]

3. Christian, P. Projection pursuit exploratory data analysis. Comput. Stat. Data Anal. 1995, 20, 669-687.

4. Zhang, C.; Dong, S. A new water quality assessment model based on projection pursuit technique. J. Environ. Sci. 2009, 21, 154-157. [CrossRef]

5. Cui, Z.Q.; Li, N. Slope Engineering: Recent Advanced in Theory and Practice; China Water \& Power Press: Beijing, China, 1999.

6. Chan, L.; Cui, H.; Li, G.; Zhang, J. Projection pursuit approach to multivariate statistical process control. Adv. Math. 2001, 30, 193-202.

7. Firedman, J.H.; Turkey, J.W. A projection pursuit algorithm for exploratory data analysis. IEEE Trans Comput. 1974, 23, 881-890. [CrossRef]

8. Kennedy, L.M.; Basu, M. Application of projection pursuit learning to boundary detection and deblurring in images. Pattern Recognit. 2000, 33, 2019-2031. [CrossRef]

9. Xiao, F.; Chen, J.G. Fractal projection pursuit classification model applied to geochemical survey data. Comput. Geosci. 2012, 45, 75-81. [CrossRef]

10. Niu, J.W.; Zhong, W.M.; Liang, Y.; Luo, N.; Qian, F. Fruit fly optimization algorithm based on differential evolution and its application on gasification process operation optimization. Knowl.-Based Syst. 2015, 88, 253-263. [CrossRef] 
11. Xu, F.; Xu, W.Y. Projection pursuit model based on particle swarm optimization for rock burst prediction. J. Geotech. Eng. 2010, 32, 718-723.

12. Wang, L.; Chen, X.; Li, Y.; Lin, K. Study on flood classification based on shuffled frog leaping algorithm and projection pursuit model. Int. J. Hydroelectr. Energy 2009, 27, 62-64.

13. Liu, D.; Zhang, G.D.; Li, H.; Fu, Q.; Li, M.; Faiz, M.A.; Alim, S.; Li, T.X.; Khan, M.I. Projection pursuit evaluation model of a regional surface water environment based on an ameliorative moth-flame optimization algorithm. Ecol. Indic. 2019, 107, 105674. [CrossRef]

14. Yu, S.; Lu, H.W. An integrated model of water resources optimization allocation based on projection pursuit model-grey wolf optimization method in a transboundary river basin. J. Hydrol. 2018, 559, 156-165. [CrossRef]

15. Zhang, X.; Ding, J.; Jin, J. Application of parametric projection pursuit regression based on genetic algorithm in flood forecasting. J. Hydraul. Eng. 2000, 31, 45-48.

16. Ni, L.; Sun, X.; Li, X.; Zhang, J. GCWOAS2: Multiobjective task scheduling strategy based on Gaussian cloud-whale optimization in cloud computing. Comput. Intell. Neurosci. 2021, 2021, 5546758. [CrossRef] [PubMed]

17. Pan, W.T. A new fruit fly optimization algorithm: Taking the financial distress model as an example. Knowl.-Based Syst. 2012, 26, 69-74. [CrossRef]

18. Tian, X.; Li, J. A novel improved fruit fly optimization algorithm for aerodynamic shape design optimization. Knowl.-Based Syst. 2019, 179, 77-91. [CrossRef]

19. Pan, Q.K.; Sang, H.Y.; Duan, J.H.; Gao, L. An improved fruit fly optimization algorithm for continuous function optimization problems. Knowl.-Based Syst. 2014, 62, 69-83. [CrossRef]

20. Yuan, X.F.; Dai, X.S.; Zhao, J.Y.; He, Q. On a novel multi-swarm fruit fly optimization algorithm and its application. Appl. Math. Comput. 2014, 233, 260-271. [CrossRef]

21. Wu, L.H.; Zuo, C.L.; Zhang, H.Q. A cloud model based fruit fly optimization algorithm. Knowl.-Based Syst. 2015, 89, 603-617. [CrossRef]

22. Wang, M.W.; Jin, J.L. The Theory and Applications of Connection Numbers; Science Press: Beijing, China, 2017.

23. Wang, M.W.; Wang, Y.; Shen, F.Q.; Jin, J.L. A novel classification approach based on integrated connection cloud model and game theory. Commun. Nonlinear Sci. Numer. Simul. 2021, 93, 105540. [CrossRef]

24. Mitić, M.; Vuković, N.; Petrović, M.; Miljković, Z. Chaotic fruit fly optimization algorithm. Knowl.-Based Syst. 2015, 89, 446-458. [CrossRef]

25. Zhang, X.; Xu, Y.T.; Yu, C.Y.; Heidari, A.A.; Li, S.M.; Chen, H.L.; Li, C.Y. Gaussian mutational chaotic fruit fly-built optimization and feature selection. Expert Syst. Appl. 2020, 141, 112976. [CrossRef]

26. Zhang, Y.W.; Cui, G.M.; Wu, J.T.; Pan, W.T.; He, Q. A novel multi-scale cooperative mutation fruit fly optimization algorithm. Knowl.-Based Syst. 2016, 114, 24-35. [CrossRef]

27. Shao, L.; Xin, Y. Safety evaluation of earth-rock dam based on projection pursuit analysis and normal cloud model. Int. J. Hydroelectr. Energy 2015, 33, 81-84.

28. Kolkiewicz, A.; Rice, G.; Xie, Y. Projection pursuit based tests of normality with functional data. J. Stat. Plan. Inference 2020, 211, 326-339. [CrossRef]

29. Peng, L.; Zhu, Q.; Lv, S.X.; Wang, L. Effective long short-term memory with fruit fly optimization algorithm for time series forecasting. Soft Comput. 2020, 24, 15059-15079. [CrossRef]

30. Xiong, C.; Lian, S. Structural damage identification based on improved fruit fly optimization algorithm. KSCE J. Civ. Eng. 2021, 25, 985-1007. [CrossRef]

31. Li, T.; Niu, W.; Ji, C. Edge user allocation by FOA in edge computing environment. J. Comput. Sci. 2021, 53, 101390. [CrossRef]

32. Liu, J.; Li, Y.; Huang, G.; Chen, L. A recourse-based type-2 fuzzy programming method for water pollution control under uncertainty. Symmetry 2017, 9, 265. [CrossRef]

33. Zeng, B.; Chen, X. Assessment of shallow landslide susceptibility using an artificial neural network. Arab. J. Geosci. 2021, 14, 499. [CrossRef]

34. Wang, H.B.; Xu, W.Y.; Xu, R.C. Slope stability evaluation using back propagation neural networks. Eng. Geol. 2005, 80, 302-315. [CrossRef]

35. Jin, J.L.; Yang, X.H.; Ding, J. Improved scheme of standard genetic algorithm-accelerated genetic algorithm. Sys. Eng. Theory Pract. 2001, 21, 8-13.

36. Wang, M.; Wang, X.; Liu, Q.; Shen, F.; Jin, J. A novel multi-dimensional cloud model coupled with connection numbers theory for evaluation of slope stability. Appl. Math. Model. 2020, 77, 426-438. [CrossRef]

37. Chen, Z.Y.; Dai, Z.H. Application of group decision-making AHP of confidence index and cloud model for rock slope stability evaluation. Comput. Geosci. 2021, 155, 104836. [CrossRef]

38. Wang, M.W.; Jin, J.L. Application of projection pursuit method to assessment of slope stability. Chin. J. Geotech. Eng. 2002, 24, 619-621. 